\title{
Geology and Composition of the Icy Galilean Satellites
}

\author{
Robert T. Pappalardo \\ Department of Geological Sciences, Box 1846, Brown University, \\ Providence, RI 02912 USA
}

\begin{abstract}
Analysis of data from the Galileo spacecraft continues to provide significant advances in our understanding of the geology and composition of Jupiter's icy Galilean satellites Callisto, Ganymede, and Europa.
\end{abstract}

\section{Callisto}

Voyager images showed that Callisto is heavily cratered at the global $(>10$ km) scale. In contrast, Galileo imaging (Fig. 1a) showed that at a small scale, Callisto's surface is remarkably crater-poor (Moore et al. 1999; Greeley, Klemaszewski, \& Wagner 2000). Instead, smooth dark material blankets the surface, with bright crater rims, central peaks, and isolated knobs poking through. The dark blanket may be global in extent. Some icy crater rims appear fragmented into isolated hills, suggesting that they eroded in place. One possibility is that sublimation is at work (Moore et al. 1999). If Callisto's surface began as a dirty ice mixture, the ice would slowly sublimate over time. Some ice would be lost to space, but some frost should be redeposited and trapped in bright, cold patches, especially at high latitudes. Over time, sublimation may disintegrate crater rims, leaving behind a dark lag deposit. Some kilometer-sized pits on Callisto may have formed by local collapse of the surface as they were undermined by gradual ice sublimation. Downslope movement of material certainly can redistribute dark material on Callisto to some extent. Galileo images show smooth dark talus derived from erosion of crater walls. Elsewhere on Callisto, tongues of dark material have streamed downhill into the crater floors (Chuang \& Greeley 2000). Some of these landslides were evidently jostled loose by nearby impacts, as small craters are found near their source regions.

High resolution spectra of Callisto's dark material obtained by the Galileo Near Infrared Mapping Spectrometer (NIMS) instrument show absorption bands indicative of clays and carbon-rich materials (McCord et al. 1997, 1998a). Absorptions at 3.4 and $4.57 \mu \mathrm{m}$ are inferred to represent $\mathrm{C}-\mathrm{H}$ and $\mathrm{C} \equiv \mathrm{N}$ stretches (respectively) of tholin-like organic materials. Absorption near $4.25 \mu \mathrm{m}$ is interpreted as $\mathrm{CO}_{2}$, and its spectral character suggests that it is trapped within or bound to dark surface materials. The $\mathrm{CO}_{2}$ absorption shows correlation to the trailing side of the satellite, suggesting a link to bombardment by corotating plasma; moreover, it is correlated with some bright impact craters (Hibbitts, McCord, \& Hansen 2000a). $\mathrm{CO}_{2}$ is a possible component of Callisto's icy subsurface, and its sublimation may contribute to generation of the satellite's dark 
surface materials as a refractory lag (Moore et al. 1999). Absorption near 4.0 $\mu \mathrm{m}$ is inferred to represent $\mathrm{SO}_{2}$ and shows a more mottled distribution, with some preference for the satellite's leading side.

Aside from tectonic features (concentric graben and scarps) associated with its large impact structures, the satellite has apparently had an uneventful tectonic and volcanic history. Callisto's multi-ringed structures, palimpsests, and scattered central dome craters hint that Callisto's ice was warm and soft enough to flow in the ancient past, but only at substantial depth. Nonetheless, Callisto has a very "young" crater-poor surface as seen at high resolution - its small craters evidently have been erased much more efficiently than on neighboring Ganymede. The mass wasting processes that have subdued and erased Callisto's topography still might be active at a slow rate today. Callisto's dearth of endogenic geological features is concordant with its only partially differentiated interior. However, Galileo magnetometer data shows strong evidence for an induced magnetic field at Callisto, implying a highly conductive interior. Surprisingly this suggests the presence of an internal briny ocean that has persisted to the present day beneath Callisto's surface.

\section{Ganymede}

Findings from high-resolution Galileo images (Fig. 1b) confirm an abundance of extensional tectonic structures within groove lanes [e.g. Pappalardo et al. 1998]. Morphological and morphometric evidence from high-resolution images, stereo-derived digital elevation models, and with Fourier analysis indicates that Ganymede's bright grooved terrain is pervasively deformed at multiple scales and suggests that both horst-and-graben and domino style normal faulting have shaped grooved terrain. A tilt-block fault geometry implies that there has been very high extensional strain in some groove lanes, an order of magnitude greater than the strain estimated from Voyager images. The high extensional strain indicated by Galileo images, along with recent laboratory data on the rheology of ice, suggests that extensional boudinage is a viable model for forming grooved terrain. Galileo observations show little direct evidence for volcanic resurfacing. Galileo results do not exclude cryovolcanism during the emplacement of grooved terrain, but grooved terrain morphology may be shaped predominantly by tectonic deformation (e.g. Prockter et al. 2000). Galileo images suggest revisions to previously inferred stratigraphy of grooved terrain, in that simple cross-cutting and truncation of older groove sets by newer ones can explain the observed stratigraphic relationships (Collins et al. 1998).

NIMS observations confirm that bright terrain is rich in $\mathrm{H}_{2} \mathrm{O}$ ice. Amorphous ice is relatively plentiful in the polar regions, where charged particle bombardment may cause redistribution of ice grains to form the polar caps (Hansen \& McCord 2000).

Ganymede's heavily cratered dark terrain comprises about one-third of Ganymede's surface. Geological investigations from Galileo high resolution images suggest that the dark material is a relatively thin lag above brighter icy material and has been affected by processes of sublimation, mass wasting, ejecta blanketing, and tectonism (Prockter et al. 2000). Dark terrain is found to be very heterogeneous in albedo at small scales, with darkest deposits occurring 

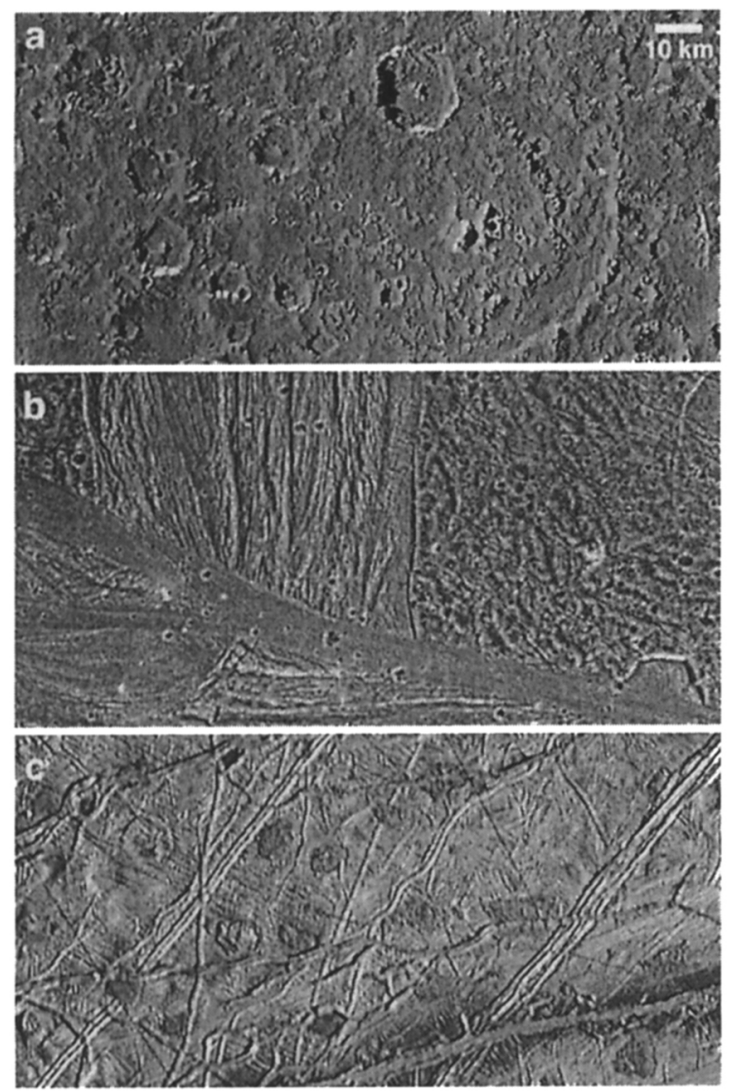

Figure 1. The three icy Galilean satellites shown to the same scale. $a$. Callisto's surface shows a range of crater sizes and degradation states and is mantled by dark debris. Kilometer-scale pits within a crater in the upper left suggest loss of subsurface volatiles and associated collapse. An 11-km crater (center left) contains a landslide of dark material. $b$. Ganymede's bright grooved terrain, such as Erech Sulcus and Sippar Sulcus, cuts older dark terrain. Extensional tectonism along with minor shear has shaped much of the grooved terrain morphology. However, it has been suggested that scalloped depressions, such as that along the boundary of Sippar Sulcus at lower right, may be sources of bright icy volcanic material that has resurfaced grooved terrain. The dark terrain of Marius Regio includes subdued furrows and more recent fractures. $c$. Europa's youthful surface is crossed by double ridges and wider bands which may have developed in response to localized heating along tidally induced fractures. Pits, domes, and spots have a preferred size of $\sim 10 \mathrm{~km}$ and may indicate solid state convection of Europa's ice shell. A liquid water ocean may lie beneath. 
within topographic lows such as craters and furrow floors. Galileo images also show pervasive tectonism contained entirely within some expanses of dark terrain. Like Callisto, NIMS observations show 3.4 , and $4.57 \mu \mathrm{m}$ absorptions in Ganymede's dark terrain, with bands about a factor of two more shallow than those on Callisto (McCord et al. 1997, 1998a). The $4.25 \mu \mathrm{m} \mathrm{CO} \mathrm{C}_{2}$ absorption shows a mottled distribution, generally correlating to darker terrain (Hibbitts et al. 2000b).

The tumultuous geological history implied by its great degree of tectonism is consistent with its highly differentiated interior in that displacement of ice polymorphs may have caused overall expansion of the satellite. The presence of a magnetic field at Ganymede and inferred molted metallic core suggests that the satellite's heating event may have occurred only about 1 billion years ago, perhaps as the result of capture into the Laplace resonance (Showman, Stevenson, \& Malhotra 1997).

\section{Europa}

Sparse craters suggest that Europa's icy surface (Fig. 1c) is young and potentially geologically active today. Dynamical modeling of potential impactors (principally Jupiter family comets) suggests a surface age of $\sim 50$ million years (Zahnle, Dones, \& Levison 1998; Pappalardo et al. 1999). This is consistent with age estimates based on estimated surface sputtering rates, and based on the lack of any detectable surface changes since the Voyager era. Thermal modelling suggests that Europa may have a subseurface ocean at relatively shallow $(\sim 20 \mathrm{~km})$ depths today, and the satellite's geology is indeed suggestive of mobile material (warm ice and/or liquid water) at shallow depth (see Pappalardo et al. 1999 for a review).

Europa's bright plains are criss-crossed by narrow troughs and enigmatic doublet ridges (paired ridges separated by a medial trough). Several models exist to explain ridges, but the most plausible is one in which localized shear heating allows warm ice to upwell along fractures (Head, Pappalardo, \& Sullivan 1999; Gaidos \& Nimmo 2000). A morphological (and hence inferred evolutionary) sequence is observed from isolated troughs to doublet ridges to wider and more complex ridge morphologies. Triple bands are ridges with ruddy diffuse margins; these dark margins may have formed through thermal alteration, cryovolcanism, and/or mass wasting. Wider pull-apart bands have formed by complete separation and spreading of the icy lithosphere, in a manner which may be broadly analogous to terrestrial sea-floor spreading.

Cross-cutting relationships indicate that Europa's dark bands brighten and fade with age. The sequence of ridge formation (inferred based on cross-cutting and color/brightness relationships) suggests that preferred ridge orientation has changed systematically over time in a means consistent with nonsynchronous rotation of the satellite's surface. This implies that the icy surface has been decoupled from the silicate interior, perhaps by an intervening liquid layer. Comparison of Voyager and Galileo images limits the current nonsynchronous rotation period to $>12000$ years. The global pattern of Europa's lineaments implies that nonsynchronous stresses and orbital flexing ("diurnal") stresses have combined to fracture Europa's icy shell. Diurnal stressing may explain Europa's extremely 
enigmatic cycloid ridge and fracture patterns, and may drive strike-slip faulting along ridges and bands. Significant tidal amplitudes are necessary to produce significant diurnal stressing, arguing for a subsurface liquid layer (Hoppa et al. 2000).

Relatively dark and red mottled terrain consists of pits, domes, dark spots, patches of smooth plains-forming material, and regions of chaos terrain. Chaos terrain is characterized by fragmented blocks of the preexisting ridged plains which have translated by as much as a few kilometers from their original positions in a dark hummocky matrix. Mottled terrain landforms suggest surface disruption along with localized partial melting. Their formation has been interpreted related to solid-state convection of the warm icy subsurface. If so, warm ice diapirs and concentrated tidal heating within them could trigger partial melting of Europa's ice shell.

Analysis of data from the Galileo NIMS instrument shows that Europa's reddish endogenic features display asymmetrical absorption bands, rather than the more symmetrical bands that characterize pure $\mathrm{H}_{2} \mathrm{O}$ ice. These asymmetric bands are interpreted as representing hydrated sulfates. McCord et al. (1998a, b) interpret the asymmetrical absorption bands as indicative of salts, notably $\mathrm{MgSO}_{4} \bullet \mathrm{XH}_{2} \mathrm{O}$, where $\mathrm{X} \geq 6$. Amorphous $\mathrm{MgSO}_{4} \bullet \mathrm{XH}_{2} \mathrm{O}$ is also a good spectral fit, suggesting rapid cooling of briny materials. Carlson, Johnson, \& Anderson (1999) infer that the hydrated material is sulfuric acid, or $\mathrm{H}_{2} \mathrm{SO}_{4} \bullet \mathrm{XH}_{2} \mathrm{O}$. If this is the case, then radiolysis can cycle Europa's surface contaminants among $\mathrm{SO}_{2}$, $\mathrm{H}_{2} \mathrm{SO}_{4}$, and sulfur chains $\left(\mathrm{S}_{x}\right)$. In this case, $\mathrm{SO}_{2}$ could explain absorption observed in the ultraviolet, and sulfur chains could account for the red contaminant observed at visible wavelengths.

\section{References}

Carlson, R., Johnson, R.E., \& Anderson, M. S. 1999, Science, 286, 97

Chuang, F. C., \& Greeley, R. 2000, JGR, 105, 20227

Collins, G. C., Head, J. W., \& Pappalardo, R. T. 1998, Icarus, 135, 345

Gaidos, E. G., \& Nimmo, F. 2000, Nature, 405, 637

Greeley, R., Klemaszewski, J. E., \& Wagner, R. 2000, Planet. Space Sci., 48, 829

Hansen, G. B., \& McCord, T. B. 2000, BAAS, 32, 1068

Head, J. W., Pappalardo, R. T., \& Sullivan, R. 1999, JGR, 104, 24223

Hibbitts, C. A., McCord, T. B., \& Hansen, G. B. 2000a, JGR, 105, 22541

Hibbitts, C. A., Pappalardo, R., McCord, T. B., \& Hansen, G. B. 2000b, Eos, in press

Hoppa, G., Tufts, B. R., Greenberg, R., \& Geissler, P. 2000, Science, 285, 1899

McCord, T. B. et al. 1997, Science, 278, 271

McCord, T. B. et al. 1998a, JGR, 103, 8603

McCord, T.B. et al. 1998b, Science, 280, 1242

Moore, J. M. et al. 1999, Icarus, 140, 294

Pappalardo, R. T. et al. 1998, Icarus, 135, 276

Pappalardo, R. T. et al. 1999, JGR, 104, 24015 
Prockter, L. M., Figueredo, P., Pappalardo, R.T., Head III, J.W., \& Collins, G. C. 2000, JGR, 105, 22519

Showman, A. P., Stevenson, D. J., \& Malhotra, R. 1997, Icarus, 129, 367

Zahnle, K., Dones, L., \& Levison, H. F. 1998, Icarus, 136, 202 